\title{
Luftverschmutzung tötet weiter
}

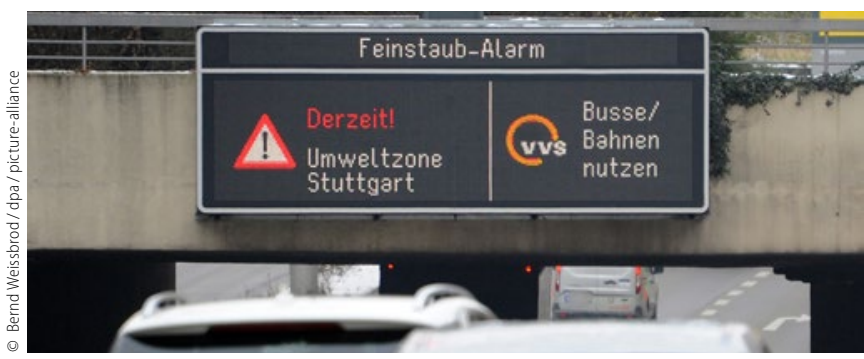

Fragestellungen: Wird durch die Ozon- und Feinstaubexposition dosisabhängig die Mortalität erhöht? Hat die Ethnizität oder der soziale Status einen Einfluss auf diese Mortalitätsursachen?

Hintergrund: Der Zusammenhang zwischen einer verminderten Lebenserwartung und der Exposition gegenüber Feinstaubpartikeln mit einer Größe unter $25 \mu \mathrm{m}$ (PM25) und Ozon ist bekannt. Die meisten Daten stammen aus Städten und aus Kollektiven mit wohlhabender Bevölkerung. Für ältere (ab dem 65. Lebensjahr) oder behinderte Bürger wurde 1965 in den USA die Krankenversicherung Medicare eingerichtet. Diese Studie untersucht die Auswirkung auf die Mortalität und Feinstaub-

Originalie

Di Q, Wang Y, Zanobetti A et al. Air Pollution and Mortality in the Medicare Population. N Engl J Med. 2016;376(26):2513-22. exposition bei Medicare-Versicherten, die in Städten oder auf dem Land wohnen.

Patienten und Methoden: Die Patientenakte von Medi-
care-Versicherten, damit $96 \%$ der gesamten Bevölkerung der USA im Alter über 65 Jahren, wurden untersucht, die Sterbedaten erhoben und die Ozon-und PM25-Konzentrationen in den jeweiligen Wohnorten der Versicherten aus Satellitendaten, meteorologischen Daten und Simulationsmodellen erhoben.

Ergebnisse: Die Studie erfasst knapp 61 Millionen Personen, die in 39.716 Orten in den USA lebten. Das Durchschnittsalter betrug 72,3 (68-82) Jahre. 44 \% der Personen waren Männer. Im Mittel wurden die Personen 7 Jahre nachverfolgt. Insgesamt 22,6 Millionen Todesfälle wurden untersucht. Die PM25-Exposition lag bei $6,21-15,64 \mu \mathrm{g} / \mathrm{m}^{3}$ und die Ozonkonzentration lag zwischen 36,27 und 55,86 ppb (parts per billion - Teile pro Milliarde). Es konnte eine 7,3\%ige Erhöhung der Mortalität mit jeder Erhöhung der PM25-Exposition von $10 \mu \mathrm{g} / \mathrm{m}^{3}$ erhoben werden (95\%-Konfidenzintervall (KI) 7,1-7,5) und für jede Erhöhung der Ozonexposition von 10 ppb eine 1,1\%ige Erhöhung der Mortalität (KI 1,0-1,2). Die Subgruppenanalysen zeigte eine erhöhte Mortalität für Männer und für die schwarze, hispanische und asiatische Bevölkerungsgruppe sowie für Personen mit niedrigem sozioökonomischem Status.

Schlussfolgerungen: Es konnte in dieser Studie eine dosisabhängige Mortalitätserhöhung bei erhöhter Exposition gegenüber PM25 und Ozon gezeigt werden sowie die Assoziation mit niedrigem sozioökonomischem Status. Überdies wurde für die schwarze Bevölkerung eine höhere Mortalität nachgewiesen. Die Senkung der Obergrenzen für die Ozon- und PM25-Belastung der Bevölkerung würde einen Gesundheitsvorteil nahelegen, der besonders wirksam ist für ethnische Minderheiten und Personen mit niedrigen Einkommen.

\section{- Kommentar Prim. Dr. Peter Dovjak und Univ.- Prof. Dr. med. Hans Jürgen Heppner}

\section{Eine unschädliche Menge Feinstaub gibt es nicht}

Wie im Editorial der gleichen Ausgabe im N Engl J Med von Berger RE et al. ausgeführt [1], gibt es keine Konzentration an Feinstaub in der Luft, die sicher niedrig genug ist, um keine negativen Gesundheitsfolgen zu haben. Die Daten aus dieser großen Studie in einer Kohorte von über 60 Millionen Medicare-Versicherten in den USA konnte eine dosisabhängige Mortalitätserhöhung für die Feinstaubbelastung zeigen. Sie

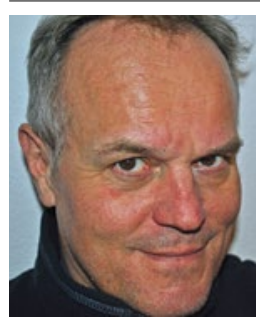

Prim. Dr. Peter Dovjak

Leiter der Akutgeriatrie

Salzkammergutklinikum Gmunden

Miller von Aichholzstraße 49

A-4810 Gmunden

peter.dovjak@gespag.at drängt auf strengere gesetzliche Regelung der Feinstaubentwicklung, trotz der Absicht des aktuelle US-Präsidenten Donald Trump, in die gegenteilige Richtung zu regulieren.
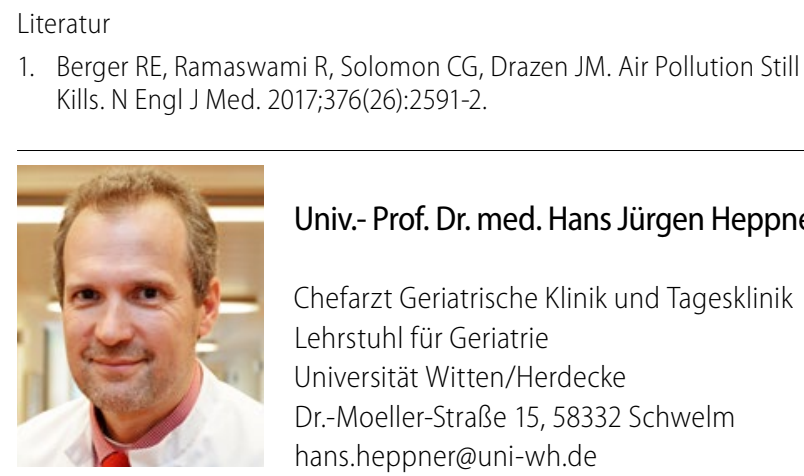

Univ.- Prof. Dr. med. Hans Jürgen Heppner

Chefarzt Geriatrische Klinik und Tagesklinik Lehrstuhl für Geriatrie Universität Witten/Herdecke Dr.-Moeller-Straße 15, 58332 Schwelm hans.heppner@uni-wh.de 